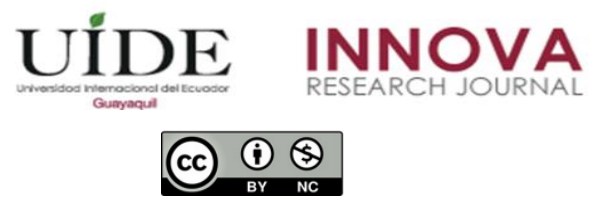

INNOVA Research Journal, ISSN 2477-9024

(Septiembre-Diciembre 2020). Vol. 5, No.3 pp. 262-272

DOI: https://doi.org/10.33890/innova.v5.n3.2020.1437

URL: http://revistas.uide.edu.ec/index.php/innova/index

Correo: innova@uide.edu.ec

\title{
Impacto de Eventos Innovadores para la Dinamización del Turismo
}

\section{Impact of Innovative Events to Boost Tourism}

María Rosa Wright Garzón

https://orcid.org/0000-0002-0745-9952

María Fernanda Montesdeoca Carrasco

https://orcid.org/0000-0003-1148-0583

Universidad Internacional del Ecuador, Ecuador

Autor por correspondencia: mawrightga@uide.edu.ec; mamontesdeocaca@uide.edu.ec

Fecha de recepción: 26 de mayo del 2020 - Fecha de aceptación: 15 de agosto del 2020

\section{Resumen}

Existen diversos motivos que generan brechas y debilidad en la competitividad del país en el turismo y en la explotación de atractivos naturales, culturales y sociales. Estos son: la ineficiente explotación de productos y servicios a escala internacional; no ver al turismo como una fuente de ingresos para la economía, la poca promoción, estrategias de bajo impacto, falta de calidad e innovación. Bajo este precepto la presente investigación tiene el objetivo de analizar el impacto de los eventos innovadores como medio para dinamizar el sector turístico en una nación, considerando que la explotación turística genera un desarrollo económico sostenible sin el perjuicio del desgaste de los recursos naturales, para lo cual se aplicó la metodología cualitativa de revisión literaria de fuentes secundarias de alto impacto, lo que garantiza la calidad de la información consultada y analizada, donde se pudo concluir que en el marco de este tipo de turismo se generan nuevas propuestas con contenidos culturales innovadores y sofisticados, donde la creación de eventos turísticos innovadores acrecentará la dinamización de destinos por medio de sus visitantes y el desarrollo económico a nivel país.

Palabras claves: turismo; eventos; dinamización; innovación; desarrollo económico

\begin{abstract}
There are various reasons that generate gaps and weakness in the country's tourist competitiveness and in the exploitation of natural, cultural and social attractions. These are: the inefficient exploitation of products and services on an international scale; not seeing tourism as a source of income for the economy, little promotion, low impact strategies, lack of quality and innovation. Under this precept, this research aims to analyze the impact of innovative events as a means to boost the tourism sector in a nation, considering that tourism exploitation generates sustainable economic development without prejudice to the wear and tear of natural resources, for which applied the qualitative methodology of literary review of high impact secondary sources, which guarantees the quality of the information consulted and analyzed, where it was concluded that within the framework of this type of tourism, new proposals are generated with innovative cultural
\end{abstract}


content and sophisticated, where the creation of innovative tourist events will increase the dynamism of destinations through its visitors and economic development at the country level.

Key words: tourism; events; dynamization; innovation; economic development

\section{Introducción}

La alta diversidad que posee Ecuador en términos culturales, paisajísticos, lugares históricos y riqueza natural son las bases en las que se potencializa su turismo. En un estudio de competitividad desarrollado por Segura (2013) determinó que la fortaleza del Ecuador en el mercado turístico internacional radica en su acervo natural, además de los diversos valores culturales que poseen sus habitantes. En tal sentido la diversidad natural y cultural juegan un papel determinante en la sostenibilidad del turismo y los beneficios económicos asociados con él. La relevancia cualitativa y cuantitativa del sector turístico que posee el Ecuador sustenta la percepción de gran potencial aprovechable.

Para Carrizo y Viera (2009) el turismo de eventos se trata de una modalidad turística donde el atractivo que genera el flujo turístico es la celebración de un evento específico. Por lo tanto, el turismo de eventos es una tipología turística de convenciones, congresos o reuniones, las cuales requieren el conocimiento de la realidad económica, social, cultural y ambiental dentro de la visión sistemática de oportunidades.

Por lo tanto, la dinamización de atractivos turísticos por medio de eventos de interés público genera un incremento en la afluencia de turistas tantos nacionales como internacionales, e incentivos al desarrollo de la economía, lo que determina la relevancia de este tipo de eventos en la dinamización turística de una nación y que su aprovechamiento puede generar muchos beneficios a este sector. Como mencionó Galindo (2011) citado por Méndez y Méndez (2018), impulsar el crecimiento económico permitirá disponer de mayor número de bienes y servicios, empleos en fin de mejorar el bienestar y el clima social para el futuro.

El viaje a un destino turístico se considera siempre como la experiencia que vive el turista, de mayor o menor significado, pero siempre es la consideración de la nueva experiencia. Un proyecto turístico debe generar experiencias nuevas y diversas al turista, fomentando en un deseo inherente de visitar nuevamente un lugar, lo que transformara dicha experiencia en desarrollo turístico y económico para una región.

Butler (1980) desarrollo el concepto de ciclo de vida de las zonas turísticas como una forma de explicar la evolución espacio-temporal de los destinos turísticos, y para comprender la dinámica asociada a la demanda de servicios turísticos, así como de sus respectivas infraestructuras. La relevancia de este estudio radica en la fluctuación de sus objetivos según la fase de desarrollo en la que el destino turístico se encuentra.

Ritchie y Hudson (2009) consideran la organización de eventos como una forma de alcanzar un posicionamiento único y diferenciado, así como una imagen de marca basada en la creación de vínculos afectivos entre el turista y el destino, yendo más allá de los atributos físicos del lugar. Con una gestión adecuada combinado con una propuesta de marketing responsable, la creación de eventos innovadores para la dinamización de atractivos turísticos puede construir una 
vía para lograr el impulso de actividades económicas, turísticas, así como la creación y consolidación de la imagen deseada de los atractivos turísticos como puntos emblemáticos de la región o ciudad.

La falta de creación de eventos innovadores por parte de las autoridades y del sector privado, como iniciativa para dar a conocer los atractivos turísticos que posee una cuidad o región, han causado un impacto negativo en el desarrollo económico de la industria hotelera, así como el de diversos negocios vinculados a esta actividad económica. El objetivo del presente trabajo es definir los diferentes beneficios de la creación de eventos innovadores para la dinamización del turismo y de la económica donde se desarrollen. De lo cual nacen las siguientes interrogantes: ¿Cómo la creación de eventos turísticos innovadores podría acrecentar la afluencia de turistas a los atractivos turísticos que posee una ciudad o región?

\section{Marco Teórico}

\section{Eventos - Turismo de Eventos}

Para Maure (2007) los eventos surgen como una exigencia por parte de la sociedad a la necesidad de asociarse en entornos geográficos, en colectivos y en grupos. Lo considera un hecho económico y cultural que permite un intercambio social, técnico, profesional y científico.

Según la definición de la Organización Mundial de Turismo (OMT), Reed Travel Exhibitions y la International Congress and Convention Association (ICCA), el término reunión hace referencia al encuentro de un grupo de personas con el objetivo de organizar o llevar a cabo una actividad concreta en dicho lugar. Estos eventos pueden ser de convenciones, conferencias, congresos, ferias comerciales, exposiciones, reuniones empresariales o de negocios, además de reuniones que cumplen con los criterios previamente mencionados (Lauman, 2014). Por lo tanto, el turismo de eventos puede ser considerado una modalidad turística donde el punto enfoque que genera el turismo como tal es la celebración de un evento.

Para Benimelis y Ordinas (2003) el turismo de eventos es un segmento de la demanda turística que al igual que la OMT incluye el turismo de convenciones, congresos y reuniones, por lo tanto, consideran la evolución del turismo de eventos presenta un desempeño notable en cuanto a los demás segmentos. Por consiguiente, este tipo de turismo se convierte en uno de los segmentos más relevantes del sector de servicios para la generar turismo como tal, permitiendo presentar como un factor transformador de la economía de un país.

Getz (1997) considero que los eventos pueden ser usados como el punto focal de atracción en torno a los cuales un destino turístico puede desarrollar una temática, imagen o atractivos específicos, logrando con esto posicionarse en la mente del turista, sin embargo, para que un destino turístico logre consolidarse como tal, es deseable que tenga uno o varios eventos de peso para que las imágenes de estos eventos y del destino turístico se hagan inseparables y se identifiquen al evento y destino como uno solo. 
El turismo de eventos puede ser utilizado como una estrategia de marketing en un destino atractivo para el viajero, que permita generar ingresos de los visitantes, productos de la actividad turística directa e indirecta que se genera en el lugar.

Entorno Turístico (2019) citando a la OMT, clasifico a 1 turismo de reuniones en cinco categorías: (a) Convenciones y Reuniones corporativas o de Negocios; (b) Congresos de Asociaciones; (c) Ferias y Exposiciones Comerciales; (d) Viajes de incentivos; y (e) Otras reuniones.

En este sentido los términos relacionados al turismo de Reuniones, tales como el turismo de negocios, convenciones, conferencias y otros se han transformado cada vez más en sectores amplios dentro del turismo de reuniones, es así como en la actualidad en el marco de la globalización industrial turística, este tipo de turismo se conoce como MICE (por sus siglas en ingles Meetings, Incentives, Conferences and Exhibitios).

Maure (2007) realizo un análisis de diferente trabajo que establecieron los principales criterios para tomar en cuenta al momento de clasificar las actividades de un evento, en la Tabla 1 se puede observar un resumen de los criterios junto con los eventos clasificados. Se destaca la clasificación de los eventos por el número de afluentes, el espacio donde estos se llevan a cabo y con mayor prioridad el motivo del evento, es por eso que el turismo de eventos busca resaltar la accesibilidad de un destino tanto para la recreación como para las actividades empresariales e incluso combinar ambas para satisfacción del viajero.

Según Gonzales y Morales (2009) los eventos comparten las características de otros productos turísticos al ser: (a) intangibles; (b) se los considera una amalgama de servicios y productos; (c) son sujeto de una demanda heterogenia y a fuertes cambios de usuarios producidos por influencias externas; (d) depende de intermediarios para su promoción y venta; y (e) no pueden ser producto estandarizados, pues aún se repetirán ya que cada evento por su desarrollo, tiempo y momento son únicos.

\section{Tabla 1}

Clasificación de eventos.

\begin{tabular}{lll}
\hline Autor & Criterio de clasificación & Eventos \\
\hline Lambín (2005) & Según su carácter o tipología & $\begin{array}{l}\text { Congreso, convención, conferencia, simposio, } \\
\text { seminario, debate, mesa redonda, taller, asamblea, } \\
\text { exposiciones y ferias, festivales, coloquios, jornada, } \\
\text { eventos multidestinos, eventos itinerantes, eventos } \\
\text { virtuales, viajes de incentivos. }\end{array}$ \\
& & $\begin{array}{l}\text { Internacionales, nacionales con participación } \\
\text { extranjera, nacionales. }\end{array}$ \\
Lambín (2005) & Por su naturaleza & $\begin{array}{l}\text { Sede captada, diseño propio. } \\
\text { Nodal (2001) }\end{array}$ \\
Maure (2006) & Según el sector generador. & $\begin{array}{l}\text { Pueden ser científico, técnicos, médicos, de ciencias } \\
\text { sociales y económicas, de agricultura y medio } \\
\text { ambiente, culturales, deportivos y náuticos, de leyes, } \\
\text { educacionales y comerciales. }\end{array}$
\end{tabular}




\begin{tabular}{lll}
\hline Autor & Criterio de clasificación & Eventos \\
\hline Herrero (2000) & Según sus objetivos & $\begin{array}{l}\text { Pueden ser promocionales; informativos, formativos- } \\
\text { didácticos, de refuerzo de relaciones sociales, de } \\
\text { relaciones internas y de relaciones externas. }\end{array}$ \\
ICCA (2005) & $\begin{array}{l}\text { Según el tamaño o número de } \\
\text { delegados. }\end{array}$ & $\begin{array}{l}\text { Mini eventos (entre 35 a 49 delegados), pequeños (de } \\
50 \text { a 249 delegados), Grandes (de 500 a 2000 } \\
\text { delegados), Mega eventos (más de 2000 participantes). }\end{array}$ \\
\hline
\end{tabular}

Fuente: Adaptación propia basada en Maure (2007).

\section{Beneficios desarrollo de eventos en el sector turístico}

El turismo de eventos es una de las ramas del sector turístico que más crecimiento ha registrado en los últimos años, convirtiéndose en una de las oportunidades de inversión de negocios más atractiva y rentable del mercado; según datos de ICCA (2005) el turismo de convenciones o eventos genera cinco veces más ingresos en la industria que el turismo de ocio y placer.

Para Benimelis y Ordinas (2003) la importancia del desarrollo del segmento del turismo de eventos se refleja en las inversiones públicas y las inversiones privadas, como son la financiación de palacios de congresos en los principales centros urbanos y las inversiones estructurales e intangibles que son importantes para mejorar la oferta. El Turismo no solo depende del destino como tal, la afluencia de turistas también dependerá del estado como tal del destino en cuanto a infraestructura y desarrollo económico de este (Ponce, 2006).

Tal como lo definió Ponce (2006), el turismo de eventos incita el crecimiento económico y territorial, que no son necesariamente coincidentes lógicos del turismo de ocio y recreación, lo que implica una diversificación de diferentes actividades económicas que podrán ser explotadas en las áreas y lugares destinados al turismo de eventos.

De la misma forma, como lo indicaron los trabajos de Faulkner (2000); Higman y Ritchie (2001); Kim y Petrick (2005); Presbury y Edwards (2005); Getz (2007); y Carrizo y Vieira (2009) quienes mencionaron que el turismo de eventos puede resultar muy beneficioso para la ciudad principal por tres motivos interrelacionados:

- La atracción de turistas y de beneficios económicos.

- La creación de infraestructura y servicios.

- La generación o consolidación de una imagen de marca para la ciudad.

Estos motivos previamente mencionados, denotan un desarrollo económico mediante la generación de infraestructura que puede ser desarrollada no solo por la empresa privada sino también con aportes del estado, ya que el ingreso de divisas y la dinamización de la economía tienen un efecto de crecimiento y prosperidad de forma directa e indirecta en una economía.

El turismo de evento puede servir para formar las artes desagradables de la ciudad (zonas marginales, zonas rurales o simplemente deterioradas por el medio ambiente o por la falta de obra pública) o generando un cambio de imagen mediante una mejora a su presentación, mediante pequeñas obras públicas como peotanización, mejora de fachadas, adecuar parques y 
lugares de recreación publica, lo cual permitirá mejorar el aspecto y la autoestima de los ciudadanos. Como indicaron Ashworth y Voogd (1990) al referirse a los gestores de la ciudad al proyectar su futuro, pueden optar por diferentes estrategias, entre las cuales mencionaron:

- Defensiva. La cual está centrada en el mantenimiento de los servicios e infraestructuras existentes en la zona.

- Orientadas a la Calidad: Esta estrategia se enfoca en mejorar las infraestructuras existentes en la zona.

- Expansionistas: Se enfoca en activar, recuperar y promocionar nuevos recursos patrimoniales de la zona de acción.

- Diversificadora: Destinada a conseguir nuevos mercados internos y externos.

\section{Dinamización del turismo}

El turismo ha alcanzado en el Ecuador un importante grado de desarrollo y consolidación, con importantes repercusiones en la estructura socioeconómica y territorial del mismo, la Industria del Turismo genera la cuarta parte de ingresos del país. La relevancia de la actividad turística es perceptible desde el punto de vista cuantitativo, por el incremento sostenido de turistas que visitan un destino, capacidad de las infraestructuras que le dan apoyo y la contrastada diversificación de productos.

Para Ivars (2003) de acuerdo con la dinámica del capitalismo internacional y las nuevas estrategias del mercado, se ha producido en los últimos años una reestructuración sin precedentes del sistema productivo y de gestión del sector, que responde a factores internos y externos. El sector de eventos en el turismo se ha marcado como una de las categorías de mayor rentabilidad, al ofrecer una propuesta combinada al viajero, quien puede disfrutar de trabajo y vacaciones al mismo tiempo, al contrario del turismo de ocio y recreación.

Considerando el caso del Ecuador, país que al ver afectada su Balanza Comercial debido a la caída del precio del petróleo, así como la caída de las remesas de los migrantes, el desarrollo turístico a nivel nacional permitió que el impacto negativo no fuera tan drástico para el país, logrando no solamente un sostenimiento de la economía, sino también logrando un desarrollo económico en zonas usualmente abandonadas por el estado.

Benites (2012) en su investigación menciono que la relación entre el turismo y el desarrollo de una zona obedece a criterios de oportunidad de la producción turística, permitiendo enlaces comerciales desde los sectores base como la agricultura hasta los sectores impulsores como el turismo.

De la misma manera Jiménez y Sánchez (2019) mencionaron que el turismo se ha desarrollado ostensiblemente en los últimos años, lo cual se puede evidenciar en el crecimiento de la competencia entre los diferentes destinos turísticos que tienen la meta de acaparar el mercado turístico, de la misma manera las empresas turísticas que han visto en el turismo de eventos una fuente fiable de ingresos se han enfocado cada vez más en este segmento del turismo, ya que se requiere de fórmulas asociativas que concentren, promuevan y promocionen dichos eventos. 
Rodríguez y San Martín (2001) y Weber (2001) hicieron referencia que, dentro de la industria turística, el turismo de eventos, reuniones y negocios representa en la actualidad uno de los segmentos de mayor atractivo y desarrollo en el contexto global, así como de forma interna, fomentando una dinamización turística interna y externa.

Los Planes de Dinamización Turística pretenden diversificar la oferta turística creando destinos de calidad alternativos a los modelos clásicos basando en la oferta cultural, gastronómica, histórica-artística, congresual, deportiva o natural de estos nuevos destinos. Los objetivos perseguidos a través de los Planes de Dinamización Turística son los siguientes:

- La puesta en valor y el uso turístico de los recursos históricos-artísticos, culturales y naturales potencialmente turísticos.

- El estudio y diseño de políticas y estrategias de productos, precio, promoción y comercialización.

- La articulación de los recursos, servicios y oferta turística como producto y su integración como destino.

- El desarrollo de una oferta de servicio turístico de calidad.

- La creación de nuevos productos turísticos basados en la exportación innovadora de recursos respetuosos con el medio ambiente.

- El fortalecimiento y la integración del tejido empresarial mediante el fomento de la asociación, asesoramiento y apoyo a las empresas.

- La integración del destino en redes que ofertan productos turísticos de características similares.

\section{Factores que incentivan el desarrollo turístico}

Fokiali, Xanthakou, Tatlidil, Tatlidil y Kaila (2006) mencionaron en su investigación que el turismo ha sido un sector en expansión en la economía de muchos países a nivel mundial, esto se debe a una serie de factores que afectan favorablemente a dicho sector, entre estos factores se puede mencionar la curiosidad que motiva al ser humano a viajar y conocer nuevos lugares, culturales y personas; de la misma forma el mejoramiento de la calidad de vida, el nivel de educación y el empleo, la mejora en los servicios de transporte en muchos países que cada vez son más seguros, eficientes y accesible para el turista, quien dispone de una diversidad de medios y precios para movilizarse.

Viñals (2002) menciono que el desarrollo de un destino turístico se debe considerar no por la afluencia de turistas que visitan dicho destino, sino por el bienestar generado por la actividad turística, es decir, el número de visitantes debe ser óptimo y correlacionado acorde a los recursos ambientales que la zona posee, la capacidad de la población local que debe organizar y orientar el desarrollo turístico y la definición de productos y servicios que se adapten a la cualificación de as necesidades de la población local y de los visitantes, quien debe ser la más beneficiada por el desarrollo de la actividad turística, considerando además los intereses de generaciones futuras. Basado en lo expresado, se plantean condiciones a considerar para lograr un óptimo desarrollo turístico, las mismas se detallan en la Tabla 2. 
Tabla 2.

Condiciones para un desarrollo turístico optimo

\begin{tabular}{ll}
\hline Condición & Descripción de la condición \\
\hline $\begin{array}{l}\text { Infraestructuras y servicios } \\
\text { generales }\end{array}$ & $\begin{array}{l}\text { Accesibilidad; trafico; transporte público; abastecimiento de agua limpia } \\
\text { y recogida de basuras; alumbrado público; asistencia sanitaria; seguridad } \\
\text { ciudadana; etc. }\end{array}$ \\
Medio ambiente & $\begin{array}{l}\text { Saturación del destino; conservación medioambiental del entorno; } \\
\text { diferente tipo de contaminación como la acústica; etc. }\end{array}$ \\
Equipos & $\begin{array}{l}\text { Equipamiento recreativo, cultural y de servicios turísticos; áreas } \\
\text { recreativas; oferta comercial, cultural y de ocio; profesionalidad de los } \\
\text { trabajadores del sector turístico y de gestión turística. }\end{array}$ \\
\hline
\end{tabular}

Fuente: Adaptación propia basada en Viñals (2002).

Saeteros Hernández, Da silva y Flores Sánchez (2019) indicaron que la sustentabilidad de las actividades económicas se vincula estrechamente con el medio ambiente, por lo tanto, la planificación turística se debe convertir en una técnica necesaria para minimizar los impactos que es turismo podría causar. Con esto en mente se debe considerar la guía de indicadores para evaluar el desarrollo sustentable de un lugar, publicado por la OMT (2004).

- Grado de satisfacción local con el turismo.

- Efecto del turismo en las comunidades locales.

- Grado de satisfacción de los turistas

- Estacionalidad del turismo

- Empleo local

- Gestión de la energía

- Disponibilidad y conservación del agua

- Calidad del agua potable

- Tratamiento de residuos (gestión de aguas residuales)

- Gestión de residuos sólidos (basura)

- Nivel de desarrollo turístico

- Grado de intensidad de uso

El desarrollo de una infraestructura adecuada para el desarrollo del turismo es de carácter vital puesto que incluye aspectos como servicios básicos, transporte, vialidad que va asegurar la seguridad y satisfacción del huésped, sin descuidar el medio ambiente y el impacto que este genere, un ejemplo es la restricción de cupos de turistas, prohibición de ingresos de animales y artículos que puedan dañar el medio ambiente y ecosistema de las Islas Galápagos.

Martín (2004) indicó que otro factor a considerar es el nivel de educación en los países receptores de turistas, convirtiéndose en una condición necesaria para el desarrollo del sector debido a que el nivel de educación de los habitantes de las zonas turísticas es directamente proporcional al nivel de los servicios turísticos prestados, razón por la cual, es relevante el considerar dicho factor al momento de diseñar un plan de desarrollo turístico optimo, en el cual debe considerarse el educar a los habitantes para brindar un buen entorno y servicio a los turistas. 


\section{Metodología}

La Metodología utilizada en el presente trabajo fue de análisis cualitativo mediante la metodología de revisión literaria, para lo cual se revisaron artículos, libros y trabajos relevantes en la academia, los cuales están indexados en revistas de alto impacto, con trabajos de alta relevancia y referenciados en diferentes trabajos. Para llevar a cabo esta investigación se realizó un Análisis-síntesis que permitió investigar la esencia del fenómeno objeto de estudio, el impacto de eventos innovadores para la dinamización del turismo, en las condiciones actuales lográndose establecer los referentes teóricos de la investigación con su base.

\section{Conclusión}

El turismo en la actualidad es una industria que dinamiza la actividad económica y motiva el desarrollo de infraestructura y preservación del medio ambiente, incentivando a las comunidades a mejorar no solo los lugares turísticos sino también a mejorar su desarrollo personal. El turismo de eventos es un segmento de la industria del turismo que se ha desarrollado y ha permitido posicionar ciudades y países en el mapa mundial, se debe considerar que en el Ecuador el desarrollo del turismo ha permitido una mejora en aspectos de infraestructura, servicios y productos turísticos, sin embargo, no se ha logrado potencializar a un nivel óptimo el turismo. La ciudad de Guayaquil, la ciudad más grande y capital económica del Ecuador, el turismo se encuentra aún en fase emergente, donde la consolidación y dinamización de un destino depende del desarrollo socioeconómico del mismo y la intervención de los profesionales del sector como comunicadores de servicios.

En este enfoque, el impacto de la creación de eventos innovadores en el sector del turismo contribuye a desestacionalizar la extrema concentración del turismo de ocio y recreación, la dinamización de un destino generara un impacto directo con efecto multiplicador en el desarrollo económico, social y cultural de la ciudad anfitriona. Todo ello supone una oportunidad de crecimiento y mejora para la ciudad anfitriona como destino de gran atractivo para la celebración de reuniones por sus infraestructuras y diversidad.

\section{Bibliografía}

Ashworth, G. \& Voodg, H. 1990. Selling the city. Marketing approaches public sector urban planning. Londres: Belhaven.

Benimelis J. y Ordinas A., (2003). El turismo de reuniones y negocios en Mallorca, Cuadernos de Turismo, Vol. 12, pp. 35-51.

Benites, (2012). Factores determinantes del desarrollo turístico del distrito de Yura, Revista TuryDes, Vol. 5 (12).

Butler, R. W. (1980). The concept of a tourist area cycles of evolution. Canadian Geographer, Vol. 24, pp. 5-12.

Carrizo, A. y Vieira, A. 2009 Turismo de eventos: desafíos estratégicos de la ciudad de João Pessoa Eugenio-Martín, J., N. Martín, y R. Scarpa (2004). "Tourism and economic growth in Latin American countries: a panel data approach". Nota di Lavoro 26. Cuadernos de Turismo, 2014 Vol. 1(23): 31-46. 
Carrizo, A. y Vieira, A. (2009). Turismo de eventos: desafíos estratégicos de la ciudad de João Pessoa (Brasil). Cuadernos de Turismo, Vol.1(23), pp: 31-46.

Entorno Turístico (2019). ¿Qué es el turismo y cómo se clasifica? Recuperado de: https://www.entornoturistico.com/que-es-el-turismo-de-reuniones-y-como-se-clasifica/

Faulkner, 2000. Monitoring the tourism impacts of the Sidney 2000 Olympics. Event Management, 6(4): 231-246.

Fokiali, P., Y. Xanthakou, R. Tatlidil, E. Tatlidil, \& M. Kaila (2006). Tourism and sustainable development strategies in Rhodes: the awareness of the local societies. Ege Academic Review, Vol. 6(2): pp. 25 - 46.

Galindo, M. (2011). Crecimiento Económico. Revista Economía ICE, 858. pp. 39 - 55.

Getz, D. (1997): Event Management \& Event Tourism. Cognizant Communication Corporation.

González, F. y Morales, S. 2009 Ciudades efímeras: Transformando el turismo urbano a través de la producción de eventos. Barcelona: Universitat Oberta de Catalunya (UOC).

Herrero, P., (2000). Gestión y Organización de Congresos. Madrid-España. Editorial Síntesis.

Higman, J. y Ritchie, B. 2001 "The evolution of festivals and other events in rural suthern New Zealand". Event Mana- gement, 7(1): 39-49.

International Congress and Convention Association [ICCA] (2005). The Association Market World Wide. Recuperado de: http://www.iccaworld.com.

Ivars, B. J. (2003). Política local y gestión de los espacios turísticos. La Geo-grafía y la gestión del turismo, págs. 17-55.

Jiménez, D. y Sánchez, M. (2019). El marketing del turismo de negocios: Los Convention Bureaux como herramienta promocional. Investigación y Marketing, 87, pp. 56-63.

Kim, S. y Petrick, J. 2005 "Residents' perceptions on impacts of FIFA 2002 World Cup: the case Of Seoul as a host city". Tourism Management, (26): 25-38.

Lauman, M. (2014). Diagnóstico estructural de la cadena de valor del turismo de reuniones en La Plata. (Tesis Maestría). Universidad Nacional de La Plata. Argentina.

Lambín, M., (2005) Informe Introductorio de base al Seminario Internacional de turismo de eventos, convenciones y negocios, Buenos Aires, Argentina.

Martín Latorre, E., San Pedro Martínez, A., Rasines Amado, L., Marsella González, E. y Sáinz De La Maza Ruíz, S., (2002): "Propuesta metodológica para la evaluación del impacto de actuaciones de los Planes de Excelencia y Dinamización Turística”, en La diversificación y la desestacionalización del sector turístico: IV Congreso de Turismo Universidad y Empresa, Tirant Lo Blanch, pp. 601 y ss.

Maure, G., (2006) Análisis y proyección del turismo de eventos en Villa Clara. (Tesis de Maestría), Universidad Central de Las Villas, Santa Clara, Cuba.

Maure, G. (2007). "Definiciones y tendencias del turismo de eventos". Contribuciones a la Economía, $N^{o} 82$.

Méndez Bravo, J. C., Quintanilla Arámbulo, E. A., \& Sánchez Jaramillo, M. E. (2018). Crecimiento económico ligado al desarrollo del emprendimiento social. INNOVA Research Journal, 3(3), 6-16. https://doi.org/10.33890/innova.v3.n3.2018.436

Ponce, M. D. (2006): «Las administraciones ante el turismo de reuniones y congresos en Murcia», Papeles de Geografía, Vol. 44, pp. 95-118.

Presbury, R y Edwards, D. 2005 "Incorporating sustainability in meetings and event Management education”. International Journal of Events Management Research, 1(1): 30-45.

Ritchie, B. y Hudson, S. (2009). Branding a memorable destination experience. The case of Brand Canada. International Journal of Tourism Research, Vol. 11(2), pp: 217-228.

Esta obra se comparte bajo la licencia Creative Common Atribución-No Comercial 4.0 International (CC BY-NC 4.0) 
Rodríguez, I. y San Martín, H. (2001). Turismo de reuniones: oportunidades de negocio. Estudios Turísticos, 147, pp. 121-139

Saeteros Hernández, A., Da Silva, E., \& Flores Sánchez, M. (2019). Turismo Sustentable y los diferentes enfoques, aproximaciones y herramientas para su medición. PASOS Revista De Turismo Y Patrimonio Cultural, 17(5), 901-914.

Segura, G. (2003). Plan de Acción Gubernamental para mejorar la Competitividad del Conglomerado de Turismo. Política de turismo del Ecuador, Vol.1, p.5

Sheng, L. 2010 "Competing or cooperating to host mega events: a simple model". Economic Modelling, (27): 375-379.

Viñals M. 2017. Turismo en espacios naturales y rurales II edit. España: Universidad Politécnica de Valencia.

Weber, K. (2001). Meeting planners' use and evaluation of convention and visitor bureaus. Tourism Management, 22(6), pp. 599-606 